\title{
Les technologies pour la santé, une discipline transversale pour la pédagogie en EEA : application aux troubles du sommeil
}

\author{
Fabienne Porée et Guy Carrault \\ fabienne.poree@univ-rennes1.fr,guy.carrault@univ-rennes1.fr \\ IUT de Rennes - Département GEII, 3 rue du Clos Courtel, BP 90422 - 35704 RENNES CEDEX 7, France \\ INSERM, U642, Rennes, F-35000, France
}

\begin{abstract}
RESUME : Les technologies pour la santé occupent aujourd'hui une place centrale dans notre société, à l'hôpital mais aussi et surtout à notre domicile. Il est donc important de former et de sensibiliser les étudiants à ces nouvelles technologies, tout en continuant à transmettre les connaissances fondamentales de l'enseignement en EEA. L'objet de cette communication est de présenter deux expériences pédagogiques différentes (l'une en TP, l'autre en projet tutoré) conduites au département GEII de l'IUT de Rennes répondant parfaitement au Programme Pédagogique National (PPN) et permettant une ouverture vers les technologies pour la santé.
\end{abstract}

Mots clés : filtrage numérique, probabilités, sommeil, Matlab, Labview, travaux pratiques, projets tutorés.

\section{INTRODUCTION}

Les technologies pour la santé occupent aujourd'hui dans notre société une place de plus en plus importante et se trouvent au carrefour de nombreuses disciplines scientifiques. Elles concernent entre autres les biocapteurs, les biomatériaux, l'image, la microchirurgie, le planning interventionnel, la télémétrie, ou le traitement du signal au sens large. L'objectif est de rendre les actes médicaux ou chirurgicaux les plus sûrs possibles. Elles s'imposent également incontournables au domicile avec l'apparition de nouveaux services aux personnes dépendantes en raison de la maladie, d'un handicap ou de l'âge. L'objectif est dans ce dernier cas de permettre une plus grande autonomie tout en garantissant une qualité de soin égale [1].

L'implication des grands instituts de recherche (INSERM, INRIA) sur ce domaine d'activité est un témoin de la dynamique qui anime cette discipline. A titre d'exemple, l'INSERM a créé un institut thématique « Technologies pour la Santé » et a étendu la notion de Centre d'Investigation Clinique au label Innovation Technologie (8 centres en France disposent d'un tel label) [2].

Sur le plan plus industriel, il convient d'appréhender la potentialité d'un tel marché. On estime que le marché mondial des technologies et des dispositifs médicaux en 2005 était de plus de 210 milliards d'euros, dont le tiers environ pour l'Europe. Le dispositif médical en Europe représente 435000 emplois dont plus de $95 \%$ relève de PME. Plus de 1500 PME sont implantées en France sur ce secteur d'activité et le poids du chiffre d'affaires représente environ $60 \%$ du chiffre des industries pharmaceutiques [3].

Cette dynamique doit donc être confortée par des formations dans ce secteur d'activités. Les plus célèbres localisées à Compiègne (UTC, spécialités Biomédical et Biomatériaux \& Biomécanique), à Grenoble (Polytech Grenoble, spécialité Technologie de l'Information pour la Santé), à Besançon (Institut Supérieur d'Ingénieur de Franche-Comté, spécialité Génie Biomédical) et maintenant à Rennes (Ecole Supérieure d'Ingénieur de Rennes, parcours Ingénierie Biomédicale) répondent parfaitement à cette exigence et cette volonté de former des ingénieurs, spécialistes en technologie pour la santé. A nos yeux, il importe également d'impulser et de sensibiliser le plus tôt possible les étudiants à ces concepts, tout en maîtrisant les connaissances de base des disciplines transversales s'y rattachant.

L'objectif de cette communication est de montrer au travers de deux exemples comment des notions très différentes et relevant de l'EEA (filtrage numérique, mathématiques appliquées) peuvent être abordées en s'appuyant sur des exemples issus de travaux de recherche en biomédical, et plus particulièrement en lien avec les troubles du sommeil [4]. Le premier exploré est construit sur le problème clinique et industriel des conséquences des troubles du sommeil sur la vigilance. Il est abordé ici très simplement en mettant en œuvre un détecteur de vigilance. Le second exemple relève des mathématiques appliquées. Il a la volonté de mettre en avant les notions fondamentales abordées dans le calcul expérimental des probabilités à partir du codage des stades de sommeil chez des nouveau-nés prématurés. Ces deux exemples sont respectivement décrits dans les sections suivantes.

\section{DETECTEUR DE VIGILANCE}

\subsection{Objectifs du TP}

Ce TP est proposé en deuxième année à l'IUT de Rennes, département GEII. Il relève du module complémentaire dit «de filtrage numérique» dont les objectifs principaux tels que définis par le Programme Pédagogique National (PPN) [5] peuvent être résumés ainsi :

- savoir réaliser la synthèse des filtres RII et RIF,

- savoir utiliser un logiciel de conception de filtres numériques,

- savoir implémenter un filtre numérique dans un processeur spécialisé,

- savoir choisir un type de filtre adapté au problème posé et savoir le réaliser.

Un choix délibéré a été pris d'inscrire un des TP dans une problématique biomédicale concrète. Son objectif est de montrer comment l'état de vigilance d'un individu (par exemple un conducteur) peut être surveillé. Ce choix est aussi intéressant puisque l'étude de la vigilance a fait l'objet de nombreuses recherches par les industriels de l'automobile à partir de différentes modalités 
d'observation telles que l'électroencéphalogramme (EEG, activité du cerveau) ou l'électromyogramme (EMG, activité musculaire).

Très classiquement, ce TP est divisé en trois parties : la préparation, le design des filtres sous Matlab et l'implémentation des filtres sous Labview.

\subsection{Préparation}

La préparation permet de s'imprégner du versant physiologique et aucune question théorique n'est posée. Elle consiste à appréhender la répartition spectrale des puissances du signal EEG en liaison avec la physiologie. En particulier, les étudiants doivent être capable d'identifier les grands rythmes de base composant l'EEG en fonction de l'état de vigilance d'un individu. Sans rentrer dans les détails, on considère que l'EEG peut être corrélé à divers états comportementaux tels que le niveau d'attention, le sommeil ou les états pathologiques. Ces états sont résumés dans le tableau 1 présentant le contenu fréquentiel du signal EEG en fonction du niveau de vigilance.

tab 1 : Répartition des différents rythmes sur les différentes bandes et états physiologiques associés, YF : yeux fermés, $Y O$ : yeux ouverts.

\begin{tabular}{|c|c|c|}
\hline Classification & $\begin{array}{c}\text { Fréquence } \\
\text { en Hertz }\end{array}$ & Niveau de Vigilance \\
\hline Rythme delta & $<4$ & $\begin{array}{c}\text { Sommeil profond } \\
\text { stades } 3 \text { et } 4\end{array}$ \\
\hline Rythme theta & $5-7$ & Somnolent stades 1 et 2 \\
\hline Rythme alpha & $8-12$ & Eveillé Calme, YF \\
\hline Rythme beta & $13-30$ & Vigilant, YO \\
\hline
\end{tabular}

Les signaux représentés sur la figure 1 illustrent les modifications observables avec la vigilance.

Outre la répartition spectrale des puissances et le lien avec la physiologie, les étudiants mesurent également la nécessité de mettre en œuvre une chaîne d'instrumentation dédiée pour recueillir des signaux de l'ordre de $100 \mu \mathrm{v}$.

\subsection{Design des filtres sous Matlab}

Le design des filtres fait directement appel à la définition du gabarit, afin de respecter des contraintes spécifiques, et au calcul de filtre.

On s'est inscrit volontairement dans une démarche très pragmatique et le calcul du filtre s'effectue directement avec le logiciel de synthèse dédié de Matlab (l'application fdatool.m cf. figure 2).

Les étudiants définissent leur gabarit et procèdent au calcul du filtre passe-bande de la bande alpha-beta (8$30 \mathrm{~Hz}$ ) et de la bande theta. Pour simplifier, ils sont construits par association de filtres passe-haut et passebas ${ }^{1}$.

1 Ceci permet aussi d'étudier l'implémentation de plusieurs cellules en cascade.

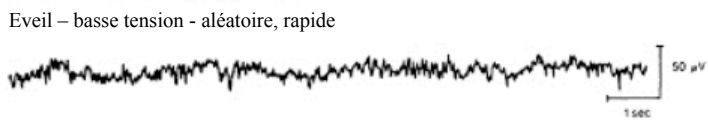

Somnolence -8 à $12 \mathrm{~Hz}$ - ondes alpha
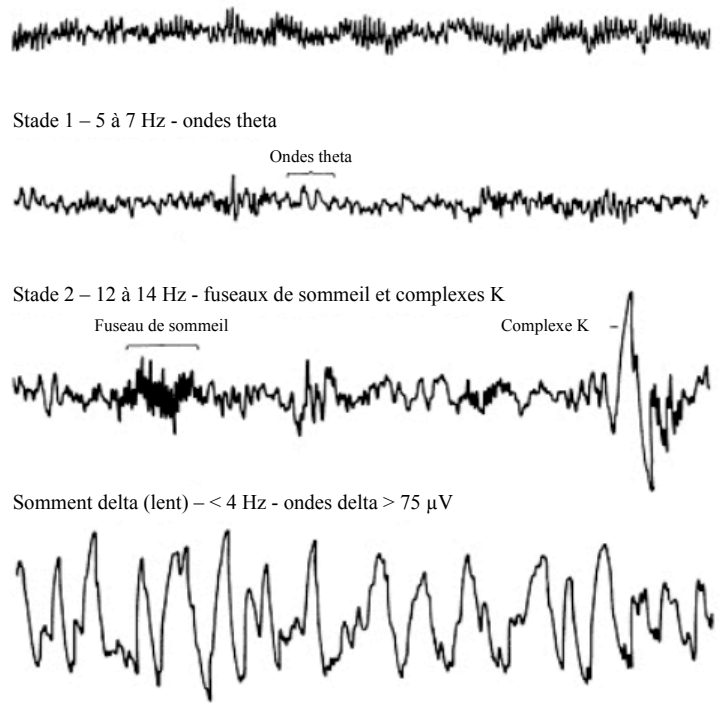

fig 1 : Exemple de signaux EEG observés (d'après le document la biologie du rêve http://membres.lycos.fr/psy3106 /PSY3106-5.pdf).

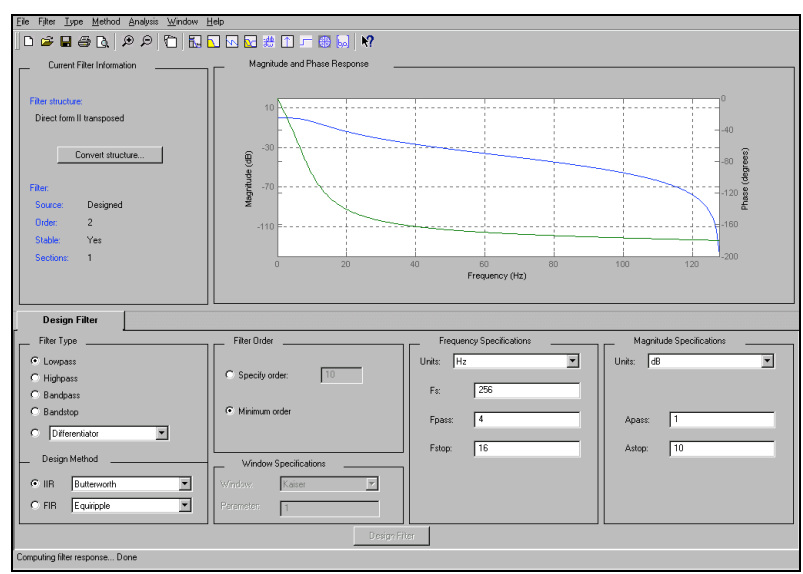

fig 2 : Interface homme-machine sous Matlab pour le design d'un filtre FIR ou IIR.

Le choix de synthétiser un filtre FIR ou IIR est laissé aux étudiants. Cependant, il est imposé d'argumenter ce choix en fonction des charges de calcul et de l'ordre du filtre $^{2}$.

Les étudiants vérifient les réponses impulsionnelles, indicielles et en fréquence des cellules élémentaires ainsi que les réponses des cellules associées.

Le taux de réjection d'une fréquence par rapport à une autre est aussi demandé.

${ }^{2}$ Cette discussion est intéressante pour l'étude de la stabilité des filtres IIR. 


\subsection{Implémentation des filtres sous Labview}

Cette ultime partie nous immerge directement dans le filtrage temps réel de données et l'élaboration d'un détecteur sous Labview. Elle sensibilise les étudiants aux notions fondamentales de récursivité et de stabilité des filtres IIR. L'implémentation du détecteur permet d'appréhender des aspects plus théoriques tels que le taux de fausse alarme ou le réglage des horizons d'observations de la fonction décisionnelle (ici le calcul d'une simple puissance dans une bande).

On considère que le signal EEG observé est échantillonné à la fréquence de $256 \mathrm{~Hz}$. La mise au point du système complet s'effectue avec un signal de synthèse n'ayant aucun mélange des bandes et permettant de tester les filtres implémentés et les fonctions de décision retenues.

Un fichier de base lire_eeg.vi est fourni et il est demandé d'intégrer :

- un graphe déroulant permettant de visualiser le signal EEG (réel) supposé acquis sur la personne,

- l'instrument virtuel permettant de ne conserver que les bandes alpha et beta,

- l'instrument virtuel permettant de ne conserver que la bande theta,

- un graphe déroulant représentant la sortie des deux filtres passe-bande,

- l'implémentation du détecteur de vigilance.

Pour ce dernier point, le signal test suppose que l'état de somnolence est caractérisé par la seule présence des ondes theta et l'état de vigilance par la seule présence des ondes alpha et beta ( $c f$. tableau 1). On admet également que les activités theta et alpha-beta ne peuvent pas être observées simultanément.

La réalisation de l'instrument virtuel qui calcule les puissances dans les deux bandes étudiées sur un horizon donné est très formatrice. Pour calculer la puissance, on retient l'estimateur de puissance suivant :

$$
P_{B .}=\frac{1}{N} \sum_{i=0}^{N} E E G^{2}(k)
$$

où $P_{B \text {. }}$ est la puissance dans la bande $B$. (alpha-beta ou theta) et $\mathrm{N}$ est un horizon d'observation, le signal $E E G(k)$ étant supposé centré. La figure 3 suivante montre le fichier initial proposé aux étudiants tandis que la figure 4 illustre un exemple d'instrument virtuel réalisé par les étudiants.

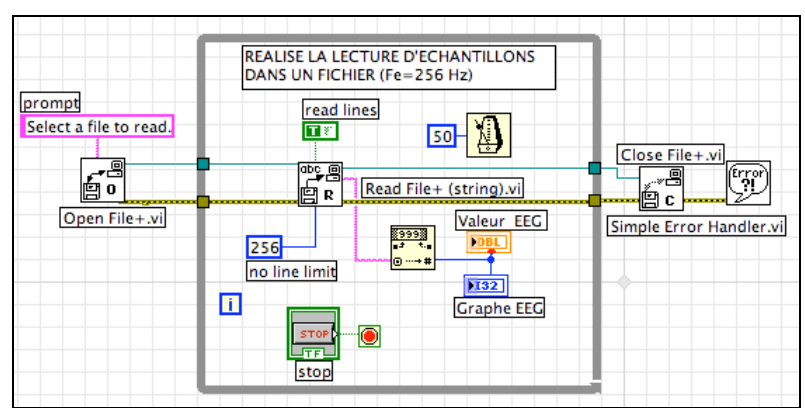

fig 3 : Diagramme initial proposé aux étudiants en début de TP. Ce dernier ne comprend que la lecture d'un fichier préenregistré inclus dans une boucle while Labview.

\subsection{Retour d'expérience}

Les sondages et les enquêtes réalisés et l'engouement que témoignent les étudiants tout au long du TP nous confortent sur le bien-fondé de notre démarche. Une synthèse de ces enquêtes est reportée dans le tableau 2. L'analyse du tableau montre que :

- les étudiants apprécient de traiter des signaux réels,

- ils sont enthousiastes quant à la démarche pratiquée (test et réalisation sur des signaux simulés et mise en œuvre sur signaux réels). Dans ce dernier cas, ils interprètent les différences observées entre les signaux tests et les signaux réels avec beaucoup d'honnêteté,

- ils démystifient complètement à la fin du TP la notion difficile -pour eux-, d'implémentation d'un filtre numérique et de mise en cascade de plusieurs cellules élémentaires. a)

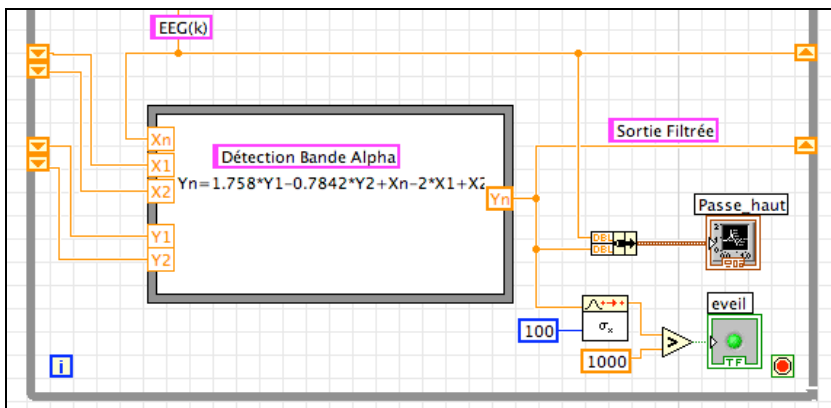

b)

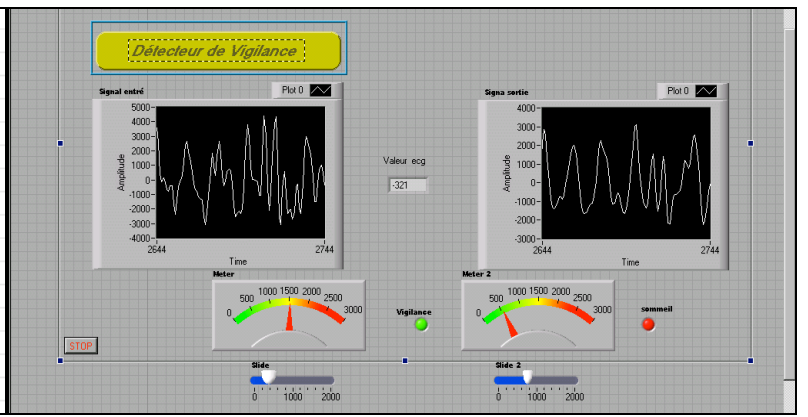
sé b) Face avant de l'instrument fig 4 : Instrument virtuel réalisé par les étudiants lors du TP a) Extrait du
virtuel réalisé 


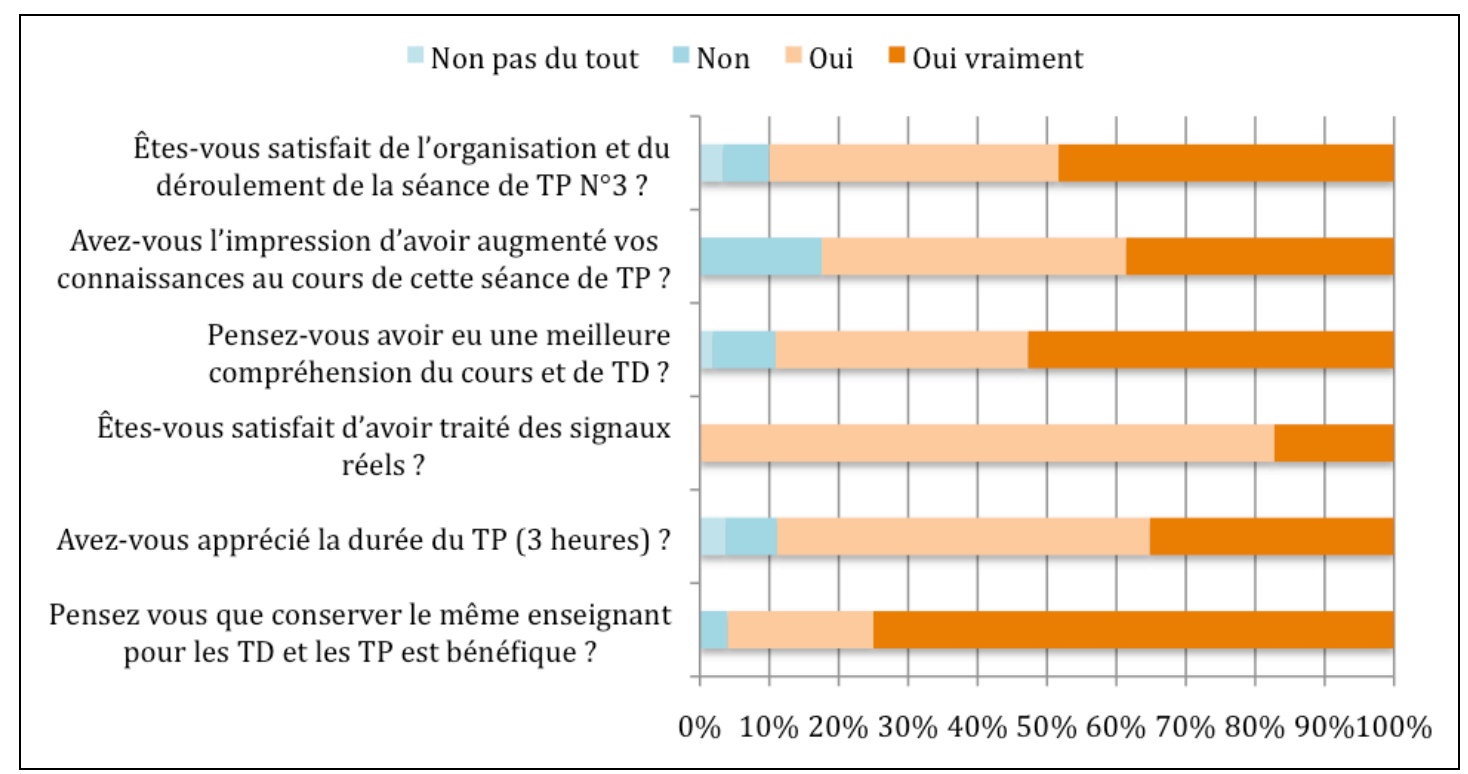

tab 2 : Extrait de l'enquête réalisée auprès des étudiants sur l'année universitaire 2009-2010 (75 étudiants ont participé à cette enquête de manière anonyme).

- $\quad$ ils sont sensibles au fait qu'ils définissent eux mêmes le gabarit du filtre.

Une question supplémentaire portant sur l'intérêt du TP a montré que les étudiants, sur les trois TP du module (l'un d'introduction, l'autre sur le traitement du son et ce troisième $\mathrm{TP}$ ), retiennent à $70 \%$ ce $\mathrm{TP}$ comme le plus intéressant.

Ces remarques positives, liées à la mise en œuvre pratique d'un filtre numérique, ne doivent cependant pas masquer que certaines difficultés subsistent sur les aspects plus théoriques.

\section{CARACTERISATION DES STADES DE SOMMEIL}

\subsection{Contexte de l'étude}

Ce travail s'inscrivait dans le cadre du projet tutoré en GEII. Succinctement, les projets tutorés, d'une durée totale de 300 heures sur les 4 semestres, ont pour objectif de placer les étudiants en situation d'autonomie [5]. Le sujet du projet a souvent pour thème une étude ayant trait aux disciplines de la spécialité sans qu'il s'agisse d'une obligation.

Ce projet doit permettre :

- l'apprentissage de la méthodologie de conduite de projet (travail en groupe, gestion du temps de travail, respect des délais, réalisation d'un cahier des charges, etc.),

- la mise en pratique des savoirs et savoir-faire (recherche documentaire, proposition de solutions, réalisation d'un rapport, etc.),

- l'apprentissage de l'autonomie,

- l'expérimentation de la transdisciplinarité.

\subsection{Les signaux}

Ce projet tutoré portait sur l'analyse de signaux de sommeil enregistrés chez des bébés grands prématurés (nés entre 29 et 31 semaines d'aménorrhée) dans une unité de soins intensifs et a été réalisé en collaboration avec le service de pédiatrie du CHU de Rennes.

Pour l'étude, des acquisitions de l'électrocardiogramme (ECG), de la respiration (Resp) et de l'électroencéphalogramme (EEG) ont été réalisées chez 15 bébés, à la fréquence de $512 \mathrm{~Hz}$, pendant des durées de 3 heures. Un exemple d'enregistrement sur une heure est reporté sur la figure 5 .

Pendant l'enregistrement des signaux, le médecin spécialiste du sommeil réalise le codage en stades de sommeil. Pour cela, il se base sur l'allure des signaux enregistrés ainsi que sur les attitudes du bébé. Cinq stades sont identifiés dans le cas des nouveau-nés [6] : le Sommeil Calme (SC), le Sommeil Indéterminé (SI), le Sommeil Agité (SA), l'Eveil Calme (EC) et l'Eveil Agité (EA). Une fois le codage terminé, on peut alors tracer un "hypnogramme», représentation en fonction du temps de l'évolution des stades de sommeil. La figure 6 montre un exemple d'hypnogramme complet obtenu pour le bébé $n^{\circ} 4$.

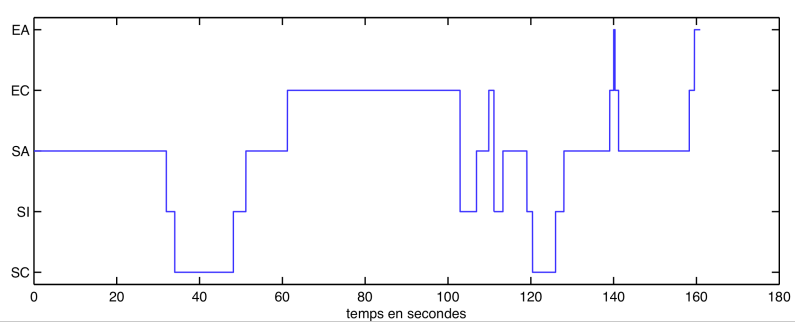

fig 6 : Hypnogramme complet du bébé $n^{\circ} 4$. 

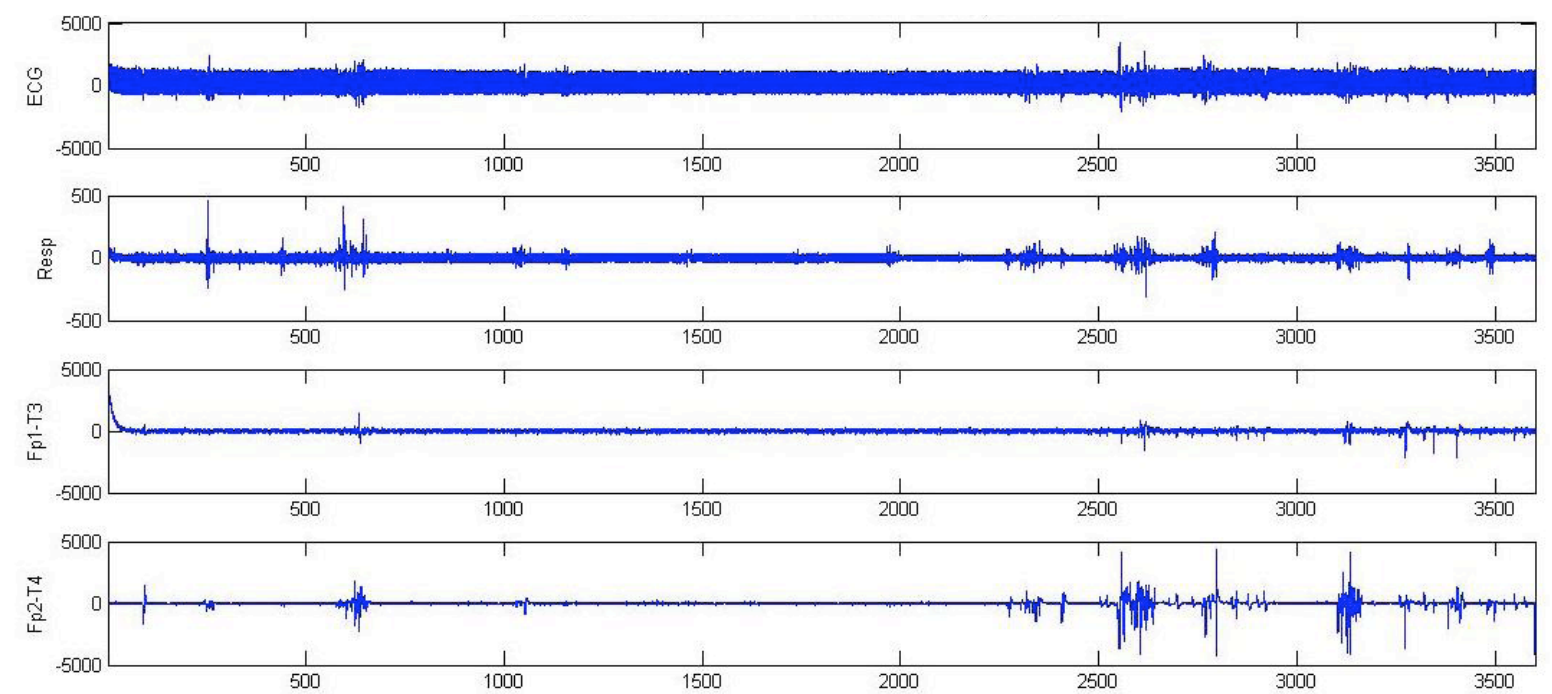

fig. 5 : Signaux enregistrés pendant le sommeil d'un nouveau-né prématuré (1 heure), ECG représente l'électrocardiogramme, Resp la respiration, Fp1-T3 et Fp2-T4 deux dérivations standards utilisées pour l'enregistrement de l'EEG [6].

\subsection{Initiation aux probabilités}

Un des objectifs du travail était de caractériser les transitions entre les différents stades de sommeil en s'appuyant sur le formalisme probabiliste. Celui retenu dans ce travail est le suivant :

- soit $Y$ la variable aléatoire discrète caractérisant le stade de sommeil dans lequel se trouve le bébé à un instant donné,

- soit $X$ la variable aléatoire discrète caractérisant le stade précédent,

$X$ et $Y$ peuvent prendre 5 valeurs : SC, SI, SA, EC ou EA. Pour chaque bébé, on peut alors calculer la probabilité d'apparition d'un stade connaissant le stade précédent, notée $P(Y=y / X=x)$, ou probabilité de transition du stade $x$ au stade $y$.

L'objet de la première partie du projet était donc d'implémenter en se basant sur les fichiers d'annotations, délivrés par l'expert, le calcul expérimental de $P(Y=y / X=x)$. A titre d'exemple, le tableau 3 donne les probabilités de transition expérimentales calculées chez le bébé $n^{\circ} 4$.

tab 3: Tableau des $P(Y=y / X=x)$ pour le bébé $n^{\circ} 4$.

\begin{tabular}{|l|c|c|c|c|c|}
\hline $\mathrm{X}$ & $\mathrm{SC}$ & $\mathrm{SI}$ & $\mathrm{SA}$ & $\mathrm{EC}$ & $\mathrm{EA}$ \\
\hline $\mathrm{SC}$ & & 1 & & & \\
\hline $\mathrm{SI}$ & 0.33 & & 0.67 & & \\
\hline $\mathrm{SA}$ & & 0.33 & & 0.67 & \\
\hline $\mathrm{EC}$ & & 0.40 & 0.20 & & 0.40 \\
\hline EA & & & & 1 & \\
\hline
\end{tabular}

Finalement, les différents tableaux obtenus sur l'ensemble de la population sont moyennés et permettent d'aboutir à un automate de profil de nuit (figure 7).

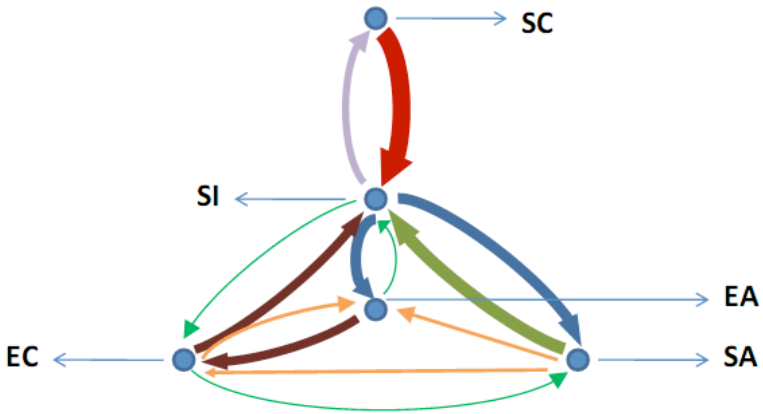

fig 7 : Automate de profil de nuit (la largeur de la flèche est proportionnelle à la probabilité de transition).

L'intérêt est ici d'établir un graphe probabiliste permettant de faire un lien avec les connaissances acquises en automatisme, sur les graphes d'état.

Sur le plan de l'application, on a pu mettre en évidence pour les 15 bébés que, par exemple :

- plusieurs transitions n'existent pas (ex : SC $\rightarrow \mathrm{SA}$ ),

- le sommeil indéterminé fonctionne comme une passerelle,

- le sommeil calme est toujours suivi par du sommeil indéterminé.

\subsection{Initiation au traitement du signal}

Dans un deuxième temps, une étude sur les signaux a été réalisée, permettant aux étudiants de transposer les notions acquises sur les variables aléatoires au domaine des signaux. L'objectif est de caractériser d'un point de vue statistique chaque segment SC, SI, SA, EC ou EA. Pour chaque stade, plusieurs paramètres ont été extraits de l'EEG : la moyenne, la médiane, la variance, la variance du signal dérivé, le kurtosis, le skewness.

Pour l'analyse des résultats, la représentation par boxplots a été utilisée car elle fournit une vision simple et 
imagée des tests statistiques. On a ainsi observé que c'était la variance de l'EEG qui permettait d'obtenir des valeurs discriminantes en fonction des stades, tout particulièrement pour les stades SC, SI et SA (figure 8). Ceci est cohérent avec la pratique, puisque ces trois stades sont identifiés par l'expert au regard de l'EEG, alors que les stades de veille (EC et EA) sont plutôt identifiés en fonction du comportement du bébé (yeux fermés ou ouverts par exemple).

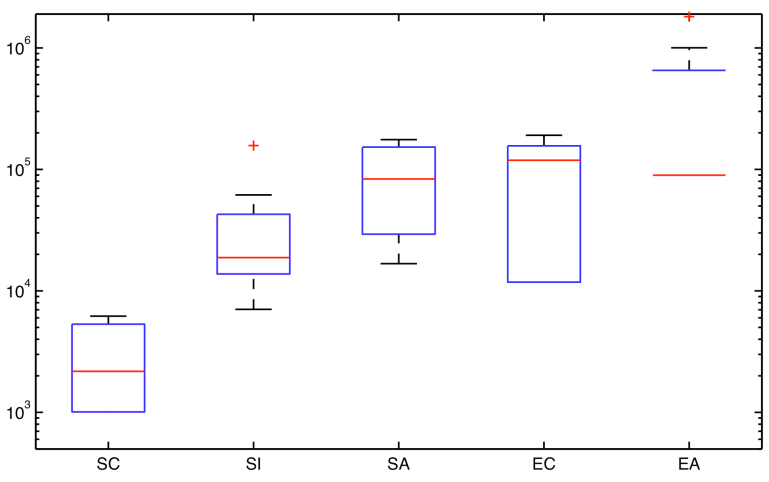

fig 8 : Boxplots obtenus avec la variance pour les différents stades de sommeil.

\subsection{Retour d'expérience}

Cette expérience nous a semblé bénéfique à différents niveaux :

- elle permet d'appliquer directement les notions, souvent complexes, de probabilités sur un exemple concret,

- une véritable réflexion est menée dans l'estimation expérimentale des probabilités,

- le calcul des différents moments statistiques des stades (moyenne, variance, kurtosis, ...) permet aux étudiants de comprendre le bien-fondé de décrire des événements par des distributions de probabilité.
Enfin, l'organisation du dernier PPN en GEII milite de plus en plus en faveur de Travaux Pratiques, même en Mathématiques. Les progrès réalisés par l'étudiant participant à ce projet nous encouragent à proposer un TP dans le module MC-M3 (Probabilités et Statistiques) sur ce sujet.

\section{CONCLUSION}

Deux exemples du domaine des technologies pour la santé appliqués au domaine de l'EEA ont été présentés dans cette communication.

Les enquêtes réalisées, dont un extrait a été reporté ici, soulignent l'intérêt et la motivation des étudiants pour ces travaux pratiques ancrés dans la réalité. Elles mettent en évidence également que les applications proposées permettent d'asseoir complètement la compréhension des cours et des TD. Leurs analyses ont permis de constater que les étudiants apprécient particulièrement d'appliquer les connaissances théoriques à des exemples concrets relevant du domaine médical.

Enfin, si l'expérience est positive et que les notions abordées semblent bien comprises, il n'en reste pas moins que certains calculs plus théoriques restent encore difficiles à maîtriser par les étudiants.

\section{Bibliographie}

[1] http://www-tecsan.cea.fr

[2] http://www.inserm.fr/index.php/thematiques/technologie -pour-la-sante/

[3] www.eucomed.be

[4] F. Porée et al., Blind source separation for ambulatory sleep recording. IEEE TITB, vol. 10, pp. 293 - 301, Avril 2006.

[5] http://media.education.gouv.fr/file/76/8/768.pdf.

[6] L.Curzi-Dascalova, M Mirmiran. Manuel des techniques d'enregistrement et d'analyse des stades de sommeil et de veille chez le prématuré et le nouveau-né à terme. Editions Inserm, Paris, 2006. 